\title{
AN EXTENSION OF THE FIRST EIGEN-TYPE AMBARZUMYAN THEOREM
}

\author{
ALP ARSLAN KIRAÇ
}

\begin{abstract}
An extension of the first eigenvalue-type Ambarzumyan's theorem are provided for arbitrary self-adjoint Sturm-Liouville differential operators.
\end{abstract}

\section{INTRODUCTION}

In 1929, Ambarzumyan [1] proved that if $\left\{(n \pi)^{2}: n \in \mathbb{N} \cup\{0\}\right\}$ is the spectrum of the boundary value problem

$$
-y^{\prime \prime}(x)+q(x) y(x)=\lambda y(x), \quad y^{\prime}(0)=y^{\prime}(1)=0
$$

with real potential $q \in L^{1}(0,1)$, then $q=0$ a.e. Clearly, if $q=0$ a.e., then the eigenvalues $\lambda_{n}=(n \pi)^{2}, n \in \mathbb{N} \cup\{0\}$.

We note that in Ambarzumyan's theorem the whole spectrum is specified. But then Freiling and Yurko [2] proved that it is enough to specify only the first eigenvalue. More precisely, they proved the following first eigenvalue-type of Ambarzumyan theorem:

Theorem 1.1. If $\lambda_{0}=\int_{0}^{1} q(x) d x$, then $q(x)=\lambda_{0}$ a.e.

Consider the boundary value problems $L(q)$ generated in the space $L^{2}(0,1)$ by the following differential equation

$$
-y^{\prime \prime}(x)+q(x) y(x)=\lambda y(x)
$$

with arbitrary self-adjoint boundary conditions, where $q \in L^{1}(0,1)$ is a real-valued function. The operator $L(q)$ is self-adjoint and its spectrum is discrete, real and bounded from below. We suppose that the eigenvalues of the operator $L(q)$ consist of the sequence $\left\{\lambda_{n}\right\}_{n \geq 1}\left(\lambda_{n} \leq \lambda_{n+1}, \lim _{n \rightarrow \infty} \lambda_{n}=+\infty\right)$ (counting with multiplicities) and $\left\{y_{n}\right\}_{n \geq 1}$ denotes the corresponding normalized eigenfunctions of the operator $L(q)$.

Let us consider another known and fixed operator $\tilde{L}:=L(\tilde{q})$ of the same domain with $L:=L(q)$ but with different potential $\tilde{q} \in L^{1}(0,1)$. Denote by $\left\{\tilde{\lambda}_{n}\right\}_{n \geq 1}$ and $\left\{\tilde{y}_{n}\right\}_{n \geq 1}$ eigenvalues and normalized eigenfunctions of the operator $\tilde{L}$, respectively.

In [3], Yurko provided the following generalization of Theorem 1.1 on wide classes of self-adjoint differential operators. Here (.,.) denotes inner product in $L^{2}(0,1)$ and $\hat{q}:=q-\tilde{q}$.

\section{Theorem 1.2. Let}

$$
\lambda_{1}-\tilde{\lambda}_{1}=\left(\hat{q} \tilde{y}_{1}, \tilde{y}_{1}\right),
$$

where $\tilde{y}_{1}$ is a normalized eigenfunction of $\tilde{L}$ related to the first eigenvalue $\tilde{\lambda}_{1}$. Then $q(x)=\tilde{q}(x)+\lambda_{1}-\tilde{\lambda}_{1}$ a.e. on $(0,1)$.

Note that the proof of this theorem is based on the well-known variation principle of the smallest eigenvalue.

Key words and phrases. Sturm-Liouville differential operators; Ambarzumyan's theorem; inverse spectral theory. 
Recently Ashrafyan [4] proved the following another generalization of the first eigenvaluetype of Ambarzumyan theorem for Sturm-Liouville problems with arbitrary self-adjoint boundary conditions.

Theorem 1.3. Let

$$
\lambda_{1}-\tilde{\lambda}_{1}=\operatorname{essinf} \hat{q} \quad \text { or } \quad \lambda_{1}-\tilde{\lambda}_{1}=\operatorname{ess} \sup \hat{q},
$$

where $\tilde{\lambda}_{1}$ is the first eigenvalue $\tilde{L}$. Then $q(x)=\tilde{q}(x)+\lambda_{1}-\tilde{\lambda}_{1}$ a.e. on $(0,1)$.

As in the proof of Theorem 1.2, the above uniqueness theorem is also provided by using the property of the smallest eigenvalue. That is, the proof of Theorem 1.3 is based on the Sturm oscillation theorem that the first eigenfunction has no zeros on interval $(0,1)$.

\section{MAin RESUlts}

The main result of this paper is as follows. Note that, to obtain the following extension theorem, it is enough to have information about the arbitrary eigenvalue $\lambda_{n}$ instead of the first eigenvalue only.

Theorem 2.1. Let, for some $n$,

$$
\lambda_{n}-\tilde{\lambda}_{n}=\left(\hat{q} \tilde{y}_{n}, \tilde{y}_{n}\right)
$$

and

$$
\lambda_{n}-\tilde{\lambda}_{n}=\operatorname{essinf} \hat{q} \quad \text { or } \quad \lambda_{n}-\tilde{\lambda}_{n}=\operatorname{ess} \sup \hat{q},
$$

where $\tilde{y}_{n}$ is a normalized eigenfunction of $\tilde{L}$ related to the eigenvalue $\tilde{\lambda}_{n}$. Then $q(x)=$ $\tilde{q}(x)+\lambda_{n}-\tilde{\lambda}_{n}$ a.e. on $(0,1)$.

Proof. It follows from the first assumption that

$$
\left(\left(\hat{q}+\tilde{\lambda}_{n}-\lambda_{n}\right) \tilde{y}_{n}, \tilde{y}_{n}\right)=0 .
$$

Since $\tilde{y}_{n}$ is a normalized eigenfunctions of the operator $\tilde{L}$ corresponding to the eigenvalue $\tilde{\lambda}_{n}$, we obtain that $\tilde{y}_{n}$ has at most finitely many isolated zero points in $(0,1)$. Therefore, the measure of the set of zero points is 0 . Hence, using this, the second assumption and $\left(\hat{q}+\tilde{\lambda}_{n}-\lambda_{n}\right) \tilde{y}_{n}^{2} \in L^{1}(0,1)$, we get $\hat{q}=\lambda_{n}-\tilde{\lambda}_{n}$ a.e. on $(0,1)$.

From Theorem 2.1, one can easily verify the following assertion.

Theorem 2.2. Let the assumptions of Theorem 2.1 be valid and let $\int_{0}^{1} q(x) d x=\int_{0}^{1} \tilde{q}(x) d x$. Then $q(x)=\tilde{q}(x)$ a.e. on $(0,1)$.

\section{ACKNOWLEDGMENTS}

This work was supported by Scientific Research Project Coordination unit of Pamukkale University numbered as 2017KKP165.

\section{REFERENCES}

1. Ambarzumian V. Über eine Frage der Eigenwerttheorie. Zeitschrift für Physik. 1929;53:690-695.

2. Freiling G, Yurko VA. Inverse SturmLiouville problems and their applications. New York: NOVA Science Publishers; 2001.

3. Yurko VA. On Ambarzumyan-type theorems. Applied Mathematics Letters. 2013;26:506-509.

4. Ashrafyan Y. A remark on Ambarzumian's theorem. Results in Mathematics. 2018 Feb;73(1):36. Available from: https://doi.org/10.1007/s00025-018-0806-9.

Department of Mathematics, Faculty of Arts and Sciences, Pamukkale University, 20070, Denizli, Turkey

E-mail address: aakirac@pau.edu.tr

Received 05.03.2020 